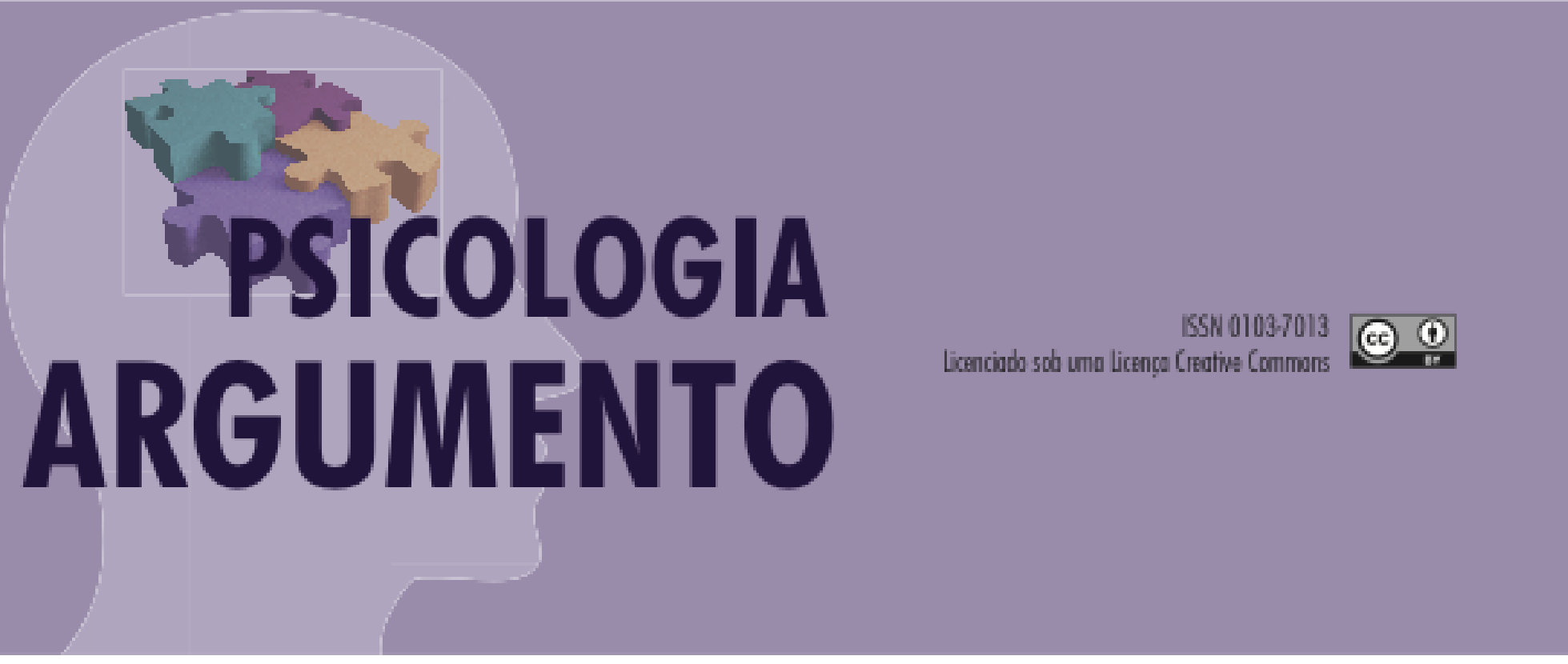

doi: 10.7213/psicol.argum.34.084.AO02

\title{
Problemas de comportamento em crianças e adolescentes com falta de controle urinário noturno
}

Behavioral problems in children and adolescents with nocturnal enuresis

Teresa Helena Schoen[a]

[a] Psicóloga. Responsável pelo atendimento psicológico no setor de Medicina do Adolescente do Departamento de Pediatria da Universidade Federal de São Paulo. São Paulo-SP, Brasil. rpetrass@uol.com.br

\section{Resumo}

A enurese é um problema que aflige muitas crianças. O objetivo deste estudo foi investigar a associação entre a falta de controle urinário noturno e problemas de comportamento. Participaram 774 pais de crianças e adolescentes de 5 a 18 anos, respondendo ao Child Behavior Checklist - CBCL. Foi selecionado o item "meu filho faz xixi na cama", que foi comparado com os demais itens do instrumento e seus resultados. Verificou-se que $8,91 \%$ dos pais assinalaram positivamente este item. Houve associação entre falta de controle urinário noturno e idade, escolaridade - os mais novos, os que estudam nas séries iniciais do Ensino Fundamental e os com defasagem idade-série escolar -, e problemas de comportamento medidos pelo CBCL. Observou-se que 'molhar a cama' é um problema comum e pode 
levar a importantes distúrbios sociais e psicológicos. A implementação de uma triagem para problemas psicológicos pode contribuir para um melhor tratamento da enurese noturna.

Palavras-chave: enurese, comportamento, comorbidade, cbcl

\begin{abstract}
Enuresis (bedwetting) is a problem that affects a large number of children. The aim of the present study was to investigate associations with bedwetting and behavioral problems. A total of 774 parents of children and adolescents aged five to 18 years responded to the Child Behavior Checklist. The item "my child wets the bed" was selected and compared to the other items on this checklist. A total of $8.91 \%$ of the parents checked the item in question. Nocturnal enuresis was associated with age (younger children), schooling (those in early years of elementary school and those behind in school for their age) and behavioral problems measured by the checklist. Bedwetting is a common problem that can lead to social and psychological disorders. The implementation of a screening protocol for psychological problems could contribute to better treatment in cases of nocturnal enuresis.
\end{abstract}

Keywords: enuresis, behavior, comorbidity, cbcl

\title{
Introdução
}

A falta de controle urinário noturno é um problema pediátrico comum, cuja prevalência varia de acordo com a idade, com a região e com a definição utilizada. $\mathrm{O}$ estudo de Byrd, Weitzman, Lanpher e Auinger (1996) encontrou que 33\% das crianças americanas ainda molhavam a cama aos cinco anos; $18 \%$ aos oito; $7 \%$ aos onze e $0,7 \%$ aos 17 anos de idade. Um estudo libanês encontrou que 7,8\% das crianças com cinco anos tinham enurese noturna; 7,5\% aos oito; $2,4 \%$ aos onze e nenhum aos 17 anos de idade (Merhi, Hammoud, Ziade, Kamel, \& Rajab, 2014). Mais meninos que meninas são afligidos por este problema (Chang, Chen, Tsai, \& Chiu, 2001; Kanaheswari, 2003; Liu, Sun, Uchiyama, Li, \& Okawa, 2000; Ozden, et al., 2007, Shereeram, He, Kalaydjian, Brothers, \& Merikangas, 2009, Solanki \& Desai, 2014). Poucas são as famílias que procuram ajuda médica para o seu tratamento (Alkot \& Deeb, 2012; Byrd et al., 1996; Kanaheswari, 2003; Lee, Sohn, Lee, Park, \& Chung, 2000, Shereeram et al., 2009, Torkashvand et al., 2015).

Dada a alta prevalência da enurese noturna (Alkot \& Deeb, 2012; Ozden et al., 2007), diversos estudos procuram verificar o impacto dessa patologia na vida das crianças e adolescentes. Há evidências crescentes sugerindo que as crianças com enurese noturna apresentam um desajuste comportamental quando comparadas com seus pares (Merhi et al., 2014). A taxa de comorbidade com transtornos comportamentais e emocionais em crianças com incontinência é significativamente maior do que em crianças continentes (20\% a 30\% das crianças com enurese noturna preenchem os critérios proposto pela CID10 ou pelo DSM IV ${ }^{\mathrm{TR}}$ para outras patologias) (Von Gontard, 2012; Von Gontard, Baeyens, Van Hoecke, Warzak, \& Bachmann, 2011).

Devido à alta taxa de comorbidade, a triagem para sintomas psicológicos é recomendada para todas as crianças que apresentam enurese noturna (Von Gontard et al, 2011). Por exemplo, no estudo de Hoecke, Fruyt, Clercq, Hoebeke e Walle (2006), utilizando o Child Behavior Checklist - CBCL (um inventário de rastreamento para 
problemas emocionais e comportamentais), foi observado que as crianças com enurese apresentaram-se significativamente com mais problemas internalizantes, externalizantes e na escala Total de Problemas. Os mesmos resultados obtiveram Alkot e Deeb (2012), além de escores mais baixos em competência escolar, e Wolfe-Christensen et al, (2014), também com problemas de atenção. Erdogan et al. (2008) encontraram associação entre enurese noturna na escala total de Problemas e no agrupamento Problemas de Sociabilidade. No estudo de Chang e Wong (2002), o grupo com enurese noturna obteve piores pontuações tanto na escala Total de Problemas e de Competência Social, quanto nos agrupamentos Problemas de Atenção, Comportamentos Agressivos e desempenho escolar.

Rocha, Emerich e Silvares (2012) observaram que crianças ou adolescentes com enurese noturna atendidos em uma clínica-escola de Psicologia apresentavam escores mais baixos em competência social, do que os outros pacientes da clínica. Koca et al (2014) encontraram escores mais altos em crianças com enurese em uma escala de depressão quando comparadas com o grupo controle. Crianças com TDAH são mais propensas do que os controles a ter enurese noturna (Burou, Avdogdu, Gurkan, Uslu, \& Sovour, 2011). No estudo de Liu et al (2000) houve associação entre enurese noturna e problemas escolares, além de comportamentais e com a adaptação social. Mau desempenho escolar também esteve associado à enurese noturna no estudo de Ozden et al. (2007).

Quase todos os estudos sobre a comorbidade entre enurese e psicopatologias em crianças sugerem uma presença maior de comportamento problemáticos nas crianças enuréticas comparadas com um grupo controle (Baeyen et al, 2005). Os resultados dependem das características do desenho do estudo e da forma como foi verificada a falta de controle urinário noturno e os problemas comportamentais. O objetivo deste estudo foi investigar a associação entre falta de controle urinário noturno e problemas de comportamento em crianças e adolescentes de uma grande cidade brasileira.

\section{Materiais e métodos}

\section{Participantes:}

Participaram deste estudo 774 pais ou responsáveis por crianças e adolescentes de 5 a 18 anos do município de São Paulo-SP, respondendo um questionário sobre seu filho.

\section{Instrumento}

Foi utilizada a versão brasileira do Child Behavior Checklist - CBCL - (1991) (Achenbach, 1991), que é um inventário de comportamentos a ser respondido por pais ou responsáveis de crianças ou adolescentes de 04 a 18 anos. As respostas devem referir-se somente aos últimos seis meses. É composto por duas partes: a primeira são sete questões a respeito de comportamentos sociais, formando a escala Total de Competência e as subescalas Competência em Atividades (5 itens), Competência Social (6 itens) e Competência Escolar (4 itens); e a segunda, 113 itens que avaliam problemas de comportamento, formando as escalas de Total de Problemas, Internalização e Externalização e oito síndromes (Ansiedade/Depressão [14 itens], Retraimento/Depressão [9 itens], Queixas Somáticas [9 itens], Problemas de Sociabilidade [8 itens], Problemas com o Pensamento [7 itens], Problemas de Atenção [11 itens], Violação de Regras [13 itens] e Comportamento Agressivo [20 itens]). A palavra síndrome refere-se aos problemas que tendem a ocorrer juntos. Alguns itens do instrumento fazem parte em mais 
de uma síndrome, enquanto outros (33) não compõem nenhuma das síndromes, fazendo parte apenas da escala Total de Problemas.

Os itens da segunda parte do CBCL são assinalados pelo responsável da seguinte forma: 0 - esta frase é falsa; 1 - esta frase é às vezes verdadeira; e 2 - esta frase é frequentemente verdadeira.

a) Dados demográficos, como sexo, idade e escolaridade (Ensino Fundamental de 8 anos) foram retirados da primeira página do instrumento.

b) Enurese noturna: $\mathrm{O}$ item 108 (faz xixi na cama) foi selecionado, para este estudo. Foram considerados com incontinência noturna a criança ou adolescente cujo responsável tenha assinalado positivamente o item (marcado a opção 1 ou 2).

c) Defasagem escolar: Defasagem escolar refere-se ao atraso na relação idade série escolar em que o aluno deveria estar cursando. Foram considerados defasados a criança ou adolescente que tivesse dois ou mais anos de atraso em relação à idade prevista para cursar a série.

d) Problemas de comportamento: foram utilizados os resultados fornecidos pelo instrumento nas escalas, subescalas e síndromes. Analisou-se também o caput dos itens de competência social e 111 itens de problemas de comportamento (ficaram de fora o item 56, pois contém 8 subitens, e o item 113, que está em branco, solicitando que o pai anote outros problemas). Para análise deste estudo, as opções 1 e 2 foram agrupadas (às vezes ou frequentemente ocorre o comportamento).

\section{Procedimento}

Coleta de dados: O Município de São Paulo-SP foi dividido em quatro regiões, e em cada região foram selecionadas três escolas (uma particular e duas públicas), um local de trabalho e um evento esportivo, totalizando doze escolas, quatro organizações e três eventos (judô, ginástica rítmica e balé). Durante o primeiro semestre de 2010, em reunião de pais e mestres, ou individualmente - no caso das empresas ou dos eventos esportivos -, os pais ou responsáveis por crianças e adolescentes de 5 a 18 anos foram convidados a responder a um questionário, podendo levá-lo para casa e entregá-lo na escola ou trabalho no prazo de uma semana. No evento esportivo, até o final da competição. Nem todos os pais abordados, aceitaram preencher o questionário; e, dos dois mil questionários distribuídos, retornaram $832(41,06 \%)$. Aqueles que descreviam crianças com menos de cinco anos ou adolescentes com mais de 18 anos e aqueles que não estavam totalmente respondidos foram excluídos, ficando o estudo com 774 questionários. Os questionários foram preenchidos de forma anônima, ou seja, sem o nome da criança ou adolescente e sem o nome dos responsáveis. Não foi solicitada a assinatura no Termo de Consentimento Livre e Esclarecido, sendo considerada a não entrega do questionário como a não aceitação de participação no estudo e vice-versa.

As respostas ao CBCL foram inseridas no programa próprio - ADM. Foi montado um banco de dados contendo as variáveis idade, sexo, a resposta dada ao item 108: "0" e "1 ou 2", os escores em cada uma das escalas e síndromes, as respostas aos outros itens de problemas de comportamento, agrupados da mesma forma: "0" e "1 ou 2", e as respostas ao caput dos itens de competência social.

Análise dos dados: Foi testada a normalidade dos dados por meio do teste de Kolmogorov-Smirnov e verificou-se que os mesmos tendem a uma distribuição normal (Teorema do Limite Central), o que garantiu o uso de testes paramétricos. Utilizou-se o 
Teste de ANOVA (comparou as médias utilizando as variâncias), de Igualdade de Duas Proporções (comparou a proporção de respostas) e Intervalo de Confiança para Média (verificou o quanto a média pode variar dentro da probabilidade de confiança).

\section{Considerações Éticas}

-...- O projeto de pesquisa que originou este estudo foi aprovado pelo Comitê de Ética em Pesquisa da Universidade Federal de São Paulo - Unifesp (CEP nº 0985/07) e autorizado pelas escolas, local de trabalho ou eventos esportivos.

\section{Resultados}

Dos 774 questionários recebidos, havia $69(8,91 \%)$ em que o item 108 estava assinalado positivamente. Destes, 39 (56,32\%) eram do sexo masculino. Embora houvesse mais meninos cujos pais informaram terem molhado a cama nos últimos seis meses, não houve diferença estatisticamente significante entre os sexos neste item $\left(X^{2}=1,00\right.$; $\mathrm{p}=0,317)$.

Houve diferença estatisticamente significante entre a faixa etária e o item 108 ter sido assinalado positivamente $(\mathrm{F}=22,76 ; \mathrm{p}<0,001)$. A média da idade para aqueles que molharam a cama nos últimos seis meses foi de 9 anos (DP 3,0), enquanto que para os que tiveram o item 108 assinalado negativamente foi de 11,1 anos (DP 3,5). A idade mínima em que o item foi assinalado positivamente foi de 5 anos e a máxima de 18 anos. Ressaltamos, porém, que nesta amostra nas idades de 15 e de 17 anos não foram encontrados indivíduos com o item 108 assinalado positivamente.

Em relação à escolaridade, houve associação $\left(X^{2}=31,44 ; \mathrm{p}<0,001\right)$ entre o ciclo e molhar a cama, sendo que mais crianças que cursavam de $1^{\text {a }}$ a $4^{\text {a }}$ série (Ensino Fundamental de 8 anos) tiveram o item 108 assinalado positivamente. Também houve associação do item com defasagem escolar $(X 2=17,16 ; p<0,001)$.

Tabela 1: Escore T médio e DP nas escalas e síndromes do CBCL, por grupo

\begin{tabular}{|c|c|c|c|c|c|c|}
\hline \multirow[t]{2}{*}{ Escalas $^{1}$} & \multicolumn{2}{|l|}{ Enurese } & \multicolumn{2}{|c|}{$\begin{array}{r}\text { Sem } \\
\text { enurese }\end{array}$} & \multirow[t]{2}{*}{$\mathbf{F}$} & \multirow[t]{2}{*}{$\mathbf{p}^{2}$} \\
\hline & Escore T & DP & Escore T & DP & & \\
\hline Ansiedade/Depressão & 63,1 & 11,4 & 58,4 & 8,2 & 19,74 & $<0,001$ \\
\hline Retraimento/Depressão & 63,9 & 11,3 & 57,9 & 8,9 & 26,97 & $<0,001$ \\
\hline Queixas Somáticas & 61,4 & 10,4 & 57,7 & 8,1 & 11,93 & 0,001 \\
\hline Problemas & 63,9 & 12,1 & 57,0 & 8,5 & 37,73 & $<0,001$ \\
\hline \multicolumn{7}{|l|}{ Sociabilidade } \\
\hline Probl com o Pensamento & 59,3 & 12,4 & 55,7 & 7,9 & 11,21 & $<0,001$ \\
\hline Problemas de Atenção & 64,3 & 11,7 & 58,2 & 9,2 & 25,35 & $<0,001$ \\
\hline Violação de Regras & 60,9 & 11,6 & 55,6 & 6,7 & 33,47 & $<0,001$ \\
\hline Comportamento Agressivo & 62,3 & 10,9 & 57,0 & 8,1 & 25,53 & $<0,001$ \\
\hline Internalização & 64,2 & 11,4 & 57,4 & 10,8 & 23,85 & $<0,001$ \\
\hline Externalização & 61,2 & 11,6 & 54,5 & 9,8 & 28,19 & $<0,001$ \\
\hline Total de Problemas & 65,7 & 12,1 & 57,1 & 10,4 & 40,63 & $<0,001$ \\
\hline
\end{tabular}




\begin{tabular}{|c|c|c|c|c|c|c|}
\hline $\begin{array}{l}\text { Competência } \quad \text { em } \\
\text { atividades }\end{array}$ & 28,0 & 17,4 & 32,2 & 17,1 & 3,64 & 0,057 \\
\hline Competência Social & 19,8 & 21,7 & 24,3 & 22,2 & 2,63 & 0,105 \\
\hline Competência Escolar & 23,5 & 22,3 & 32,9 & 23,5 & 9,44 & 0,002 \\
\hline Total de Competências & 26,8 & 17,1 & 31,8 & 17,8 & 4,58 & 0,033 \\
\hline
\end{tabular}

${ }^{1}$ Nas escalas e síndromes de problemas de comportamento, quanto maior o escore, mais problemas a criança apresenta. Nas escalas de Competência Social, quanto menor o escore, menos competência a criança apresenta.

Em relação aos problemas de comportamento, foram encontradas diferenças estatisticamente significativas em todas as escalas e síndromes (Tabela 1). Entretanto, em relação à competência social, somente em duas subescalas. A Tabela 1 apresenta o T escore médio obtido pelas crianças e adolescentes que tiveram o item 108 assinalado positivamente, comparando-o com o $\mathrm{T}$ escore médio daqueles cujos pais informaram ser falsa essa afirmação.

Tabela 2: Associação entre o item 108 (faz xixi na cama) e os itens que compõem a escala de Internalização.

\begin{tabular}{|c|c|c|c|c|c|}
\hline Item $^{1}$ & $\mathrm{Z}$ & $\mathrm{p}$ & Item* & $\mathrm{Z}$ & $\mathrm{p}^{2}$ \\
\hline 12. solidão & 0,83 & 0,407 & 14. chora muito & 4,63 & $<0,001$ \\
\hline $\begin{array}{l}\text { 31. medo de fazer algo } \\
\text { ruim }\end{array}$ & 1,53 & 0,126 & $\begin{array}{l}\text { 32. tem que ser } \\
\text { perfeito }\end{array}$ & 0,15 & 0,879 \\
\hline 33. ninguém gosta dele & 3,32 & $<0,001$ & $\begin{array}{l}\text { 34. Sente-se } \\
\text { perseguido }\end{array}$ & 2,70 & 0,007 \\
\hline 35. desvalorizado & 2,28 & 0,023 & $\begin{array}{l}\text { 42. prefere ficar } \\
\text { sozinho }\end{array}$ & 0,09 & 0,928 \\
\hline 45. nervoso & 1,27 & 0,205 & 50. medroso & 2,30 & 0,022 \\
\hline 51. tonturas & 0,34 & 0,732 & 52. muito culpado & 1,26 & 0,209 \\
\hline 54. cansaço & 1,73 & 0.084 & 65. recusa-se a falar & 4,36 & $<0,001$ \\
\hline 69. reservado & 2,06 & 0,039 & 71. embaraçado & 1,27 & 0,203 \\
\hline 75. tímido & 1,37 & 0,171 & 80. olhar parado & 2,08 & 0,038 \\
\hline 88. emburrado & 2,42 & 0,015 & 89. desconfiado & 2,19 & 0,028 \\
\hline 102. falta energia & 4,16 & $<0,001$ & 103. triste & 2,65 & 0,008 \\
\hline 111. retraído & 2,54 & 0,011 & 112. preocupado & 0,50 & 0,614 \\
\hline
\end{tabular}


Em relação aos itens do instrumento, a maioria apresentou uma associação estatisticamente significante com o item 108, ou seja, quem molhou a cama nos últimos seis meses, também teve outros problemas de comportamento assinalados (Tabelas 2 a 5 ). Os dois itens (tem alergia e tem asma) que não fazem parte da escala Total de Problemas, não apresentaram associação com enurese noturna.

Tabela 3: Associação entre o item 108 (faz xixi na cama) e os itens que compõem a escala de Externalização.

\begin{tabular}{|c|c|c|c|c|c|}
\hline Item $^{1}$ & $\mathrm{Z}$ & $\mathrm{p}^{2}$ & Item $^{1}$ & $\mathrm{Z}$ & $\mathrm{p}^{2}$ \\
\hline 3. discute muito & 1,36 & 0,173 & 7. convencido & 0,31 & 0,753 \\
\hline 16. maldoso & 4,80 & $<0,001$ & 19. exige atenção & 1,99 & 0,046 \\
\hline 20. destrói suas coisas & 5,02 & $<0,001$ & 21. destrói dos outros & 4,25 & $<0,001$ \\
\hline 22. desob. em casa & 2,28 & 0,023 & 23. desob. na escola & 5,61 & $<0,001$ \\
\hline 26. não sente culpa & 1,36 & 0,174 & 27. ciúmes & 3,80 & $<0,001$ \\
\hline 37. briga & 3,54 & $<0,001$ & 39. más companhias & 4,37 & $<0,001$ \\
\hline 43. mente & 3,02 & 0,003 & 57. ataca fisicamente & 5,43 & $<0,001$ \\
\hline 63. prefere os mais & 1,38 & 0,167 & 67. foge de casa & 3,72 & $<0,001$ \\
\hline \multicolumn{6}{|l|}{ velhos } \\
\hline 68. grita muito & 2,00 & 0,046 & 72. põe fogo & 3,71 & $<0,001$ \\
\hline 74. palhaçadas & 2,84 & 0,004 & 81. rouba em casa & 5,35 & $<0,001$ \\
\hline 82. rouba fora de casa & 6,70 & $<0,001$ & 86. mal humorado & 0,66 & 0,512 \\
\hline 87. mudanças de humor & 1,24 & 0,214 & 90. xinga & 2,40 & 0,016 \\
\hline 93. fala muito & 1,33 & 0,185 & 94. goza dos outros & 3,41 & $<0,001$ \\
\hline 95. esquentado & 3,34 & $<0,001$ & 96. pensa muito em & 1,12 & 0,264 \\
\hline & & & sexo & & \\
\hline 97. ameaça as pessoas & 5,82 & $<0,001$ & 101. mata aula & 3,66 & $<0,001$ \\
\hline 104. barulhento & 2,82 & 0,005 & 105. drogas/álcool & 4,94 & $<0,001$ \\
\hline 106. vandalismo & 6,39 & $<0,001$ & & & \\
\hline
\end{tabular}

${ }^{1}$ simplificação do enunciado do item ${ }^{2}$ teste de Igualdade de Duas Proporções 
Tabela 4: Associação entre o item 108 (faz xixi na cama) e alguns dos itens que compõem as síndromes Problemas com o Pensamento e Problemas com a atenção

\begin{tabular}{|c|c|c|c|c|c|}
\hline Item $^{1}$ & $\mathrm{Z}$ & $\mathrm{p}^{2}$ & Item $^{1}$ & $\mathrm{Z}$ & $\mathrm{p}^{2}$ \\
\hline 1. comp. infantil & 4,36 & $<0,001$ & 8. concentração & 2,36 & 0,018 \\
\hline 9. obsessões & 0,73 & 0,468 & 10. inquieto & 3,13 & 0,002 \\
\hline 11. dependente & 3,24 & $<0,001$ & 13. confuso & 2,15 & 0,031 \\
\hline 17. mundo da lua & 1,54 & 0,125 & 25. dificul. com pares & 4,41 & $<0,001$ \\
\hline 38. alvo de gozação & 3,00 & 0,003 & 40. ouve sons & 2,68 & 0,007 \\
\hline 41. impulsivo & 2,21 & 0,027 & 46. tiques & 2,91 & 0,004 \\
\hline 48. não gostam dele & 4,53 & $<0,001$ & 55. pesa muito & 2,12 & 0,034 \\
\hline 61. mal na escola & 3,98 & $<0,001$ & 62. desajeitado & 4,64 & $<0,001$ \\
\hline 64. prefere mais novos & 1,66 & 0,098 & 66. compulsões & 2,88 & 0,004 \\
\hline 70. vê coisas & 3,98 & $<0,001$ & 84. comp. estranho & 2,79 & 0,005 \\
\hline 85. pensam. estranhos & 4,02 & $<0,001$ & & & \\
\hline
\end{tabular}

Tabela 5: Associação entre o item 108 (faz xixi na cama) e os itens que só fazem parte da Escala Total de Problemas

\begin{tabular}{|c|c|c|c|c|c|}
\hline Item $^{1}$ & $\mathrm{Z}$ & $\mathrm{p}^{2}$ & Item $^{1}$ & $\mathrm{Z}$ & $\mathrm{p}^{2}$ \\
\hline $\begin{array}{l}\text { 5. age como sexo } \\
\text { oposto }\end{array}$ & 2,87 & 0,004 & 6. fora do vaso sanitário & 7,65 & $<0,001$ \\
\hline 15.cruel com animais & 5,16 & $<0,001$ & $\begin{array}{l}\text { 18. machuca-se de } \\
\text { propósito }\end{array}$ & 4,53 & $<0,001$ \\
\hline 24. não come bem & 2,51 & 0,012 & 28. come o que não serve & 2,40 & 0,016 \\
\hline 29. medos & 1,87 & 0,062 & 30. medo da escola & 6,75 & $<0,001$ \\
\hline $\begin{array}{l}\text { 36. machuca-se } \\
\text { frequente. }\end{array}$ & 4,37 & $<0,001$ & 44. rói unhas & 2,26 & 0,024 \\
\hline 47. pesadelos & 1,32 & 0,187 & 49. constipado & 0,52 & 0,603 \\
\hline 53. come muito & 2,21 & 0,027 & 58. cutuca a pele & 1,67 & 0,004 \\
\hline 59. masturb. em públ. & 2,91 & 0,004 & 60. excesso de masturb. & 2,69 & 0,007 \\
\hline 73. probl. sexuais & 4,98 & $<0,001$ & 76. dorme menos & 0,07 & 0,946 \\
\hline 77. dorme mais & 1,95 & 0,052 & 78. brinca com fezes & 2,89 & 0,004 \\
\hline 79. probl. de fala & 4,66 & $<0,001$ & 83. junta coisas & 3,26 & $<0,001$ \\
\hline 91. ideação suicida & 3,31 & $<0,001$ & 92. fala/anda dormindo & 1,31 & 0,189 \\
\hline 98. chupa dedo & 3,86 & $<0,001$ & 99. preoc. com limpeza & 0,77 & 0,440 \\
\hline 100. prob. com sono & 1,78 & 0,074 & 107. urina-se de dia & 8,05 & $<0,001$ \\
\hline 109. choraminga & 4,39 & $<0,001$ & 110. ser do sexo oposto & 5,95 & $<0,001$ \\
\hline
\end{tabular}


Os itens de competência social, somente os referentes à escola apresentaram associação com o item 108: já repetiu de série escolar $\left(X^{2}=3,853 ; p=0,050\right)$ e apresenta problemas acadêmicos $\left(X^{2}=5,256 ; \mathrm{p}=0,022\right)$.

\section{Discussão}

O objetivo deste estudo foi investigar a associação entre a falta de controle urinário noturno (aqui medido pelo item 108 do CBCL) e a presença de problemas de comportamento em crianças e adolescentes (utilizando o mesmo instrumento).

Diferentemente da literatura, embora semelhante aos estudos de Mota, Barros, Matijasevich e Santos (2015), e de Salih (2012), não houve associação entre enurese noturna e sexo masculino.

A prevalência de crianças/adolescentes que molham a cama foi de $8,91 \%$, mais alta que em crianças chinesas (4,3\%, 6-16 anos) [Liu et al, 2000] ou de Taiwan (6,8\%, 6-11 anos) [Chang et al, 2001] ou da Malásia (8\%, 7-12 anos) [Kanaheswari, 2003]; mas mais baixa (mesmo com um critério mais elástico adotado neste estudo) que crianças egípcias (11,5\%, 6-18 anos) [Alkot \& Deeb, 2012], eslovenas (12,5\%, 6-15 anos) [Karničnik, Koren, Kos \& Varda, 2012], da Turkia (17,5\%, 6-12 anos) [Ozden et al., 2007], ou mesmo de Juiz de Fora-MG (15,87\%, 6-14 anos) [Dahan, 2015]. Provavelmente questões culturais influenciam a tolerância dos pais em relação à enurese noturna. Países orientais costumam iniciar o treino da toalete em idades mais jovens que países ocidentais (Liu et al, 2000). Também a utilização de fraldas descartáveis, ou dormir em cama/quarto sozinho são fatores que levam alguns pais a adiarem o treino da toalete, deixando o controle urinário somente por conta da maturação da criança. A faixa etária estudada neste trabalho foi mais ampla que em outros, abrangendo adolescentes mais velhos, o que pode ter gerado uma porcentagem total menor de indivíduos que molham a cama.

Estudos anteriores demonstraram que a prevalência da enurese tende a diminuir com a idade (Byrd et al., 1996; Ozden et al, 2007). Semelhantemente, o presente estudo também observou a diminuição da porcentagem de pais que assinalaram positivamente o item 108 com o avanço da idade de seus filhos. Isto pode ser devido à busca de tratamento (pouco provável, como mostram Alkot e Deeb [2012] ou Lee et al. [2000]), resolução espontânea (provavelmente se aplica apenas para aqueles com sintomas leves - poucas molhadas [Oguz et al., 2015,]) ou ao aprendizado de acordar a noite para ir ao banheiro. Em nossa prática clínica, quando os pais são inquiridos a respeito de enurese noturna, muitos deles informam que demoraram a ter o controle, entretanto hoje não mais molham a cama, mas levantam-se à noite, ou seja, aprenderam a contornar o problema. Diversos estudos mostram que isto ocorre, como, por exemplo, o de Goessaert et al. (2015). Uma das limitações deste estudo foi o baixo número de adolescentes de 17 e 18 anos (21 em cada faixa etária), o que dificulta descobrir a prevalência de incontinência urinária em adolescentes mais velhos.

Nossos resultados mostraram que a falta de controle urinário esteve associada com um risco significativamente aumentado de problemas comportamentais, emocionais, sociais e acadêmicos, o que vai ao encontro de estudos anteriores que detectaram impactos negativos sobre a saúde mental de crianças ou adolescentes com enurese noturna (Byrd et al., 1996; Chang \& Wong, 2002; Erdogan et al., 2008; Zink, Freitag, \& Von Gontard, 2008). É importante ressaltar que o item 108 do CBCL não compõe nenhuma das síndromes ou subescalas. Se fizesse parte, seria esperada uma associação intrínseca; como, 
na construção do instrumento, este item não fez parte de nenhum dos constructos de padrões de problemas de comportamento, podemos considerar que a falta de controle urinário está associada aos problemas de comportamento ou competência social por outros motivos.

Von Gontard (2012) observou que quatro hipóteses para esta associação são possíveis. Os sintomas psicológicos, emocionais e escolares podem (1) ser uma consequência da falta de controle urinário noturno; (2) ser a causa; (3) ser devidos a fatores etiológicos comuns; e (4) podem coocorrer por acaso. Em relação à primeira hipótese mais estudos consideram uma consequência. Provavelmente, crianças com enurese noturna podem experimentar castigos físicos, humilhação ou isolamento social, que levam a um grande medo do que pode ocorrer quando da detecção da cama molhada, por exemplo. Esse estresse psicológico que acompanha as tentativas dos pais ou responsáveis de desenvolver o controle urinário do filho ou descarregar sua própria frustação pelos transtornos que ele causa pode aumentar o risco de problemas comportamentais e emocionais. Nesse sentido, os problemas aqui detectados como associados à enurese noturna seriam decorrentes dela, talvez um comportamento de enfrentamento para lidar com as consequências negativas da enurese no contexto familiar.

Em relação à segunda hipótese, os problemas levam à enurese, eventos estressores (por exemplo, o divórcio dos pais) ou distúrbios comportamentais (TDHA, por exemplo) podem preceder e induzir a enurese. O presente estudo não observou estas variáveis, embora tenha visto associação entre Problemas com a Atenção e o item 108 ter sido assinalado positivamente.

Outra explicação (terceira hipótese) para esta associação seria que a enurese noturna e os problemas comportamentais ou emocionais compartilhem as mesmas causas biológicas, sociais e psicológicas. As atitudes dos responsáveis frente ao treino da toalete e controle urinário podem ser semelhantes às atitudes frente a outras demandas do desenvolvimento. Corroborando esta hipótese está o estudo de Fergusson e Horwood (1994), onde se observou que, embora o sexo da criança, sua maturidade social, seu QI, o nível socioeconômico da família, estresse familiar e/ou conflito parental estivessem associados às taxas de problemas de comportamento, após o ajuste desses fatores, as crianças que molhavam a cama após a idade de 10 anos mostraram ligeiros aumentos nas taxas de problemas comportamentais e déficit de atenção até a idade de 13 anos e as taxas de aumento de ansiedade até a idade de 15 anos. Baeyens, Roeyers, Van Erdeghem, Hoebeke e Walle (2007) observaram, em um estudo de seguimento, que muitas crianças continuaram preenchendo os critérios para o TDAH, mesmo depois de pararem de molhar a cama, sugerindo a presença de uma síndrome psiquiátrica, em vez de um comportamento de enfrentamento. Touchette et al. (2005) verificaram que havia uma associação entre enurese (ou atraso no controle para aqueles que não apresentavam mais a incontinência noturna) e atrasos nos marcos de desenvolvimento na primeira infância, como aquisição da linguagem, da marcha ou outras habilidades motoras. Esses autores defendem a ideia de que molhar a cama pode ser um indicativo de um possível atraso no desenvolvimento do sistema nervoso central. O presente estudo não traz evidências a respeito desta hipótese.

Embora diversos estudos sobre problemas comportamentais/emocionais/escolares pudessem descartar a quarta hipótese - do acaso -, alguns outros não conseguiram comprovar uma associação entre enurese e os problemas apresentados. O fato de alguns estudos não obterem associação entre enurese noturna e determinados problemas 
comportamentais (por exemplo, Chang \& Wong, 2002, ou Erdogan et al., 2008) mostra que as associações são complexas e diferem para cada subtipo individual de desordem, e como os fatores genéticos e ambientais podem interagir (Zink et al, 2008). Deve-se levar em consideração os subtipos de enurese (primária ou secundária, monossintomática ou não), a severidade (número de molhadas semanais), presença de incontinência diurna, além de outras variáveis. O presente estudo mostrou forte associação entre presença de problemas de comportamento, emocionais, sociais e escolares com a enurese noturna, entretanto, em alguns itens não houve esta associação, como em outros estudos (item 4 [Dahan, 2015]). Portanto, este trabalho não consegue descartar esta hipótese.

A maioria dos estudos nos leva a pensar que a primeira hipótese (a enurese noturna e os acontecimentos decorrentes dela, como surras, humilhações, levariam ao desenvolvimento de problemas psicológicos) seja verdadeira. Para Erdogan et al. (2008) e Ozden et al (2007), a associação entre os escores de problemas de comportamento e enurese refletem diferenças nas atitudes dos pais em relação às crianças com ou sem enurese noturna. O trabalho de Theunis, Van Hoecke, Paesbrugge, Hoebeke e Walle (2002) deixa claro que os problemas por eles estudados (autoimagem, aceitação social, competência escolar e física, comportamento e autoestima) ocorreram como decorrência da enurese. Embora este estudo não permita estipular qual a causa e qual a consequência, estamos mais propensos a considerar que grande parte dos sintomas apresentados pelas crianças/adolescentes com falta de controle urinário noturno seja decorrente do manejo deste problema. A associação estatisticamente significante com quase todos os itens do instrumento, nos levam a pensar que o mais provável é que a enurese cause outros problemas, e não que a existência desses leve a criança a desenvolver incontinência vesical.

Em relação à associação do item 108 com os demais, observa-se, também, apoio na literatura. Por exemplo, o item 06 (faz suas necessidades fora do banheiro) vai ao encontro do estudo de Zink et al. (2008) ou o item 61 (seus trabalhos escolares são fracos), com o estudo de Liu et al (2000). Houve coocorrência entre molhar a cama e outros problemas de comportamento.

\section{Considerações finais}

Este é um estudo transversal, e como é verdade para toda a pesquisa transversal, não se pode demonstrar a direção causal entre enurese noturna e problemas comportamentais. Embora o instrumento tenha sido preenchido por pais ou responsáveis em diferentes locais da cidade de São Paulo-SP, dependeu da autorização destes locais, o que dificulta a randomização do estudo. Observou-se um número alto de não devolução do instrumento. Pode ser que pais, cujos filhos não apresentam problemas quanto a este marco do desenvolvimento, não tenham se interessado em responder ao instrumento. Há uma diminuição da frequência de pais de adolescentes, tanto nas reuniões escolares (frequência maior de pais cujos filhos apresentem problemas escolares), quanto acompanhando seus filhos em eventos esportivos. Talvez este tenha sido o motivo de haver menos protocolos, neste estudo, de indivíduos mais velhos, o que dificulta tanto a identificação da frequência de enurese noturna nesta faixa etária, quanto à verificação de associação entre enurese noturna e problemas comportamentais. Outra limitação do presente estudo é a falta de informações detalhadas sobre a enurese noturna, tendo-se baseado apenas no preenchimento de um item de um instrumento de triagem. Embora não 
tendo sido específico, os resultados deste estudo têm implicações importantes para uma compreensão mais ampla da enurese noturna, bem como pode subsidiar o saber do profissional que precisa orientar os pais de crianças com falta de controle urinário noturno sobre os problemas comportamentais, emocionais, sociais e acadêmicos que possivelmente estarão presentes.

O presente estudo verificou que $8,91 \%$ dos pais assinalaram como verdadeiro o item 108: "meu filho faz xixi na cama". Não houve associação entre a falta de controle urinário noturno e o sexo, mas houve em relação à idade e a escolaridade: os mais novos, aqueles que estudam nas séries iniciais do Ensino Fundamental e aqueles que já repetiram alguma série escolar. Houve forte associação entre enurese noturna e os problemas de comportamento medidos pelo CBCL.

A enurese é um problema comum entre crianças e adolescentes e está associada a importantes distúrbios sociais e psicológicos. A falta de controle urinário noturno quando este já deveria ter ocorrido deve ser uma condição que leve os profissionais de saúde a ampliar sua investigação, explorando questões comportamentais, emocionais, sociais e escolares em maior profundidade. A implementação de uma triagem para problemas emocionais e comportamentais e apoio psicológico podem contribuir para um melhor tratamento da enurese.

\section{Referências}

Achenbach, T. M. (1991). Integrative Guide for the 1991 CBCL/4-18, YSR and TRF Profiles. Burlington, VT: University of Vermont Department of Psychiatry.

Alkot, M., \& Deeb, M. (2012). Nocturnal Enuresis among school children in Menofia governorate, Egypt: a hidden problem. Journal of American Science, 8(1), 327-334.

Baeyens, D., Roeyers, H., Van Erdeghem, S., Hoebeke, P., \& Walle, J. (2007). The prevalence of attention deficit-hyperactivity disorder in children with nonmonosymptomatic nocturnal enuresis: a 4-year follow up study. Journal of Urology, 178(6), 2616-2620.

Burou, B., Aydogdu, O., Gurkan, K., Uslu, R., \& Sovour, T. (2011). Lower urinary tract conditions in children with attention deficit hyperactivity disorder: correlation of symptoms based on validated scoring systems. Journal of Urology, 185(2), 663-668.

Byrd, R. S., Weitzman, M., Lanphear, N. E., \& Auinger, P. (1996). Bed-wetting in US children: epidemiology and related behavior problems. Pediatrics, 98(3), 414-419.

Chang, P., Chen, W. J., Tsai, W. Y., \& Chiu, Y. N. (2001). An epidemiological study of nocturnal enuresis in Taiwanese children. British Journal of Urology International, 87, 678-681.

Chang, S. S., \& Wong, S. N. (2002). Behavioural problems in children and parenting stress associated with primary nocturnal enuresis in Hong Kong. Acta Paediatrica, 91(4), 475-479.

Dahan, P. (2015). Associação entre asma e enurese em população escolar de Juiz de Fora. Dissertação de Mestrado não-publicada, Faculdade de Medicina, Universidade Federal de Juiz de Fora, Juiz de Fora-MG. 
Erdogan, A., Akkurt, H., Boettjer, N. K., Yurtseven, E., Can, G., \& Kiran, S. (2008). Prevalence and behavioural correlates of enuresis in young children. Journal of Paediatrics and Child Health, 44(5), 297-301.

Fergusson, D.M. \& Horwood, L.J. (1994). Nocturnal enuresis and behavioral problems in adolescence: a 15-year longitudinal study. Pediatrics, 94(5), 662-668.

Goessaert, A. S., Schoenaers, B., Opdenakker, O., Hoebeke, P., Everaert, K., Walle, J. V. (2015). Long-term followup of children with Nocturnal Enuresis: Increased frequency of Nocturia in adulthood. Pediatric Urology, 192(6), 1893-1895.

Hoecke, E. V., Fruyt, F. D., Clercq, B. D., Hoebeke, P. \& Walle, J. V. (2006). Internalizing and Externalizing Problem Behavior in Children with Nocturnal and Diurnal Enuresis: A Five-Factor Model Perspective. Journal of Pediatric Psychology, 31(5), 460-468.

Kanaheswari, Y. (2003). Epidemiology of childhood nocturnal enuresis in Malaysia. Journal of Paediatric and Child Health, 39(2), 118-123.

Karničnik, K., Koren, A., Kos, N., \& Varda, N. M. (2012). Prevalence and quality of life of Slovenian children with Primary Nocturnal Enuresis. International Journal of Nephrology, 27(9), 1740-1741.

Koca, O., Akyüz, M., Karaman, B., Özcan, Z., Öztürk, M. Sertkaya, Z., et al. (2014). Evaluation of depression and self-esteem in children with monosymptomatic nocturnal enuresis: A controlled trial. Archivio Italiano di Urologia e Andrologia, 86(3), 212-214.

Lee, S. D., Sohn, D. W., Lee, J. Z., Park, N. C., \& Chung, M. K. (2000). An epidemiological study of enuresis in Korean children. BJU International, 85(7), 869-873.

Liu, X., Sun, Z., Uchiyama, M., Li, Y., \& Okawa, M. (2000). Attaining nocturnal urinary control, nocturnal enuresis, and behavioral problems in Chinese children aged 6 through 16 years. Journal of the American Academy of Child and Adolescent Psychiatry, 39(12), 1557-1564.

Merhi, B., Hammoud, A., Ziade, F., Kamel, R., \& Rajab, M. (2014). Mono-Symptomatic Nocturnal Enuresis in Lebanese Children: Prevalence, Relation with Obesity, and Psychological Effect. Clinical Medicine Insights: Pediatrics, 8, 5-9.

Mota, D., Barros, A., Matijasevich, A., \& Santos, I. (2015). Prevalence of enuresis and urinary symptoms at age 7 years in the 2004 birth cohort from Pelotas, Brazil. Jornal de Pediatria, 91(1), 52-58.

Oguz, U. Aykac, A., Demirelli, E., Sancak, E., Resorlu, B., Sarikaya, S., et al. (2015). The time of spontaneous resolution of Monosymptomatic Nocturnal Enuresis (MNE) is familial.Urologia Internationalis, 94(4), 459-463.

Ozden, C., Ozdal, O. , Altinova, S., Oguzulgen, I., Urgancioglu, G., \& Memis, A. (2007). Prevalence and associated factors of enuresis in turkish children. International Brazilian Journal of Urology, 33(2), 216-222. 
Rey, J. M., Bird, K. D., \& Hensley, V. R. (1995). Bedwetting and psychopathology in adolescentes. Journal of Paediatric and Child Health, 31(6), 508-512.

Rocha, M., Emerich, D., \& Silvares, E. (2012). Perfil comportamental de crianças e adolescentes encaminhados para atendimento por enurese ou outras queixas. Psico PUCRS, 43(1), 77-84.

Salih, A. A. (2012). Nocturnal enuresis: Prevalence and associated factors. A sample of children in Baghdad. World Family Medicine Journal, 10(5), 29-32.

Shreeram, S., He, J. P., Kalaydjian, A., Brothers, S., \& Merikangas, K. R. (2009). Prevalence of enuresis and its association with attention-deficit/hyperactivity disorder among U.S. children: results from a nationally representative study. Journal of the American Academy of Child and Adolesceny Psychiatry, 48(1), 35-41.

Solanki, A. \& Desai, S. (2014). Prevalence and risk factors of nocturnal enuresis among school age children in rural areas. International Journal of Research in Medical Sciences, 2(1), 202-205.

Theunis, M., Van Hoecke, E., Paesbrugge, S., Hoebeke, P., \& Walle, J. (2002). Self-image and performance in children with nocturnal enuresis. European Urology, 41(6), 660-667.

Torkashvand, F., Rezaeian, M., Bagheani, T., Dawarani, M., Zarafshan, H., Mostafavi, S, et al. (2015). Prevalence of nocturnal enuresis in school-age children in Rafsanjan. Journal of Pediatric Nephrology, 3(2), 71-74.

Touchette, E., Petit, D., Paquet, J., Tremblay, R. E., Boivin, M., \& Montplaisir, J. Y. (2005). Bed-wetting and its association with developmental milestones in early childhood. Archives of Pediatrics \& Adolescent Medicine, 159(12), 1129-1134.

Von Gontard A. (2012). Does psychological stress affect LUT function in children? ICI-RS 2011 Neurourology and Dynamics, 31(3), 244-248. doi: 10.1002/nau.22216

Von Gontard, A., Baeyens, D., Van Hoecke, E., Warzak, W. J, \& Bachmann, C. J.(2011). Psychological and psychiatric issues in urinary and fecal incontinence. Journal of Urology, 185(4), 1432-1436. doi: 10.1016/j.juro.2010.11.051

Wolfe-Christensen, C., Fedele, D., Grant, D., Veenstra, A., Kovacevic, L., Elder, J., et al. (2014). Factor Analysis of the Pediatric Symptom Checklist in a Population of Children with Voiding Dysfunction and/or Nocturnal Enuresis. Journal of Clinical Psychology in Medical Settings, 21(1), 72-80.

Zink, S., Freitag, C., \& von Gontard, A. (2008). Behavioral comorbidity differs in subtypes of enuresis and urinary incontinence. Journal of Urology, 179(1), 295-298.

Recebido / Received: 29/08/2016

Aprovado / Approved: 16/10/2016 